\title{
Tendencias de la morbilidad y letalidad hospitalaria por cáncer
}

\author{
H. GUANCHE GARCELL, E. GARCÍA ARZOLA, J. GONZÁLEZ ISLA, \\ E. SALOMÓN ZALDIVAR, P. PÉREZ MONTERO \\ Departamento de Epidemiología Hospitalaria. Hospital Universitario "Joaquín \\ Albarrán”. La Habana. Cuba
}

CANCER TRENDS IN HOSPITAL MORBIDITY AND LETHALITY

\begin{abstract}
RESUMEN
Objetivo: Describir la tendencia en la morbilidad y letalidad por cáncer en el Hospital Universitario “Joaquín Albarrán” (La Habana, Cuba).

Método: Realizamos un estudio de series temporales de los ingresos por cáncer (enero 1999-diciembre 2005). Se obtuvo la información de los ingresos totales y por cáncer, las defunciones, y las tasas de letalidad (fallecidos por cáncer/ingresos por cáncer x 100). De los ingresos ocurridos durante el 2005 se registró la edad (media y desviación standard), sexo, la causa de la admisión, y se precisó el momento del diagnóstico de cáncer.

Resultados: Se demuestra un sostenido incremento de la proporción de ingresos por cáncer, con la menor de 3\% (año 1999) y la mayor de $7,99 \%$ (año 2005). Las tasas de letalidad tuvieron un comportamiento irregular con la menor en el $1999(12,7 \%)$ y la mayor en el 2001 $(27,86 \%)$. El servicio de medicina interna atendió la mayoría de los pacientes $(60,71 \%)$, seguidos por cirugía general $(26,81 \%)$. Al $44 \%$ de los pacientes se les diagnosticó cáncer durante el ingreso, mientras que el $56 \%$ se diagnóstico previamente, de los cuales el $42,1 \%$ ingresaron para tratamiento (quirúrgico o quimioterapia antineoplásica) y el 53,5\% por complicaciones propias del proceso maligno.

Conclusión: Se ha demostrado una tendencia ascendente en la morbilidad hospitalaria por cáncer, lo que obliga a modificar las estrategias de atención de estos pacientes que aseguren la calidad de los servicios ante el incremento de la demanda.
\end{abstract}

PALABRAS CLAVES: Cáncer. Morbilidad. Letalidad. Atención hospitalaria. Cuidados paliativos.

\section{ABSTRACT}

Objetive: Describe trends in morbidity and lethality of cancer in University Hospital "Joaquín Albarrán” (La Habana, Cuba).

Method: Carry out a temporal series studies of patients admited from january 1999 to december 2005. We obtain informationa about admission, total and for cancer, deceased patients and letality rates (deceased for cancer/admission for cancer x 100). From the 2005's admission we obtain age, sex, cause of admission, and if the diagnostic of cancer was doing during this admission or before.

Results: Trend of cancer's admision have continuous increase during 1999-2005 period, with 3\% of admission in 1999 to $7.99 \%$ in 2005. Lethality rates have an irregular behavior with smaller rate in 1999 (12.7\%) and bigger in 2001 (27.86\%). Internal Medicine service gave care to $60.71 \%$ of cancer admission, with less frecuency in general surgery service (26.81\%). $44 \%$ of patients were diagnosis during this admission, and $56 \%$ the diagnosis was doing in previous admission, of wich $42.1 \%$ were admited to treatment (surgical and drugs) and $53.5 \%$ for cancer complications.

Conclusions: We show a continuous increased trend in hospital cancer morbidity. It is a commit to modify healthcare's strategies of cancer patient adressed to guarante the quality of services in front of the incresed demand.

KEY WORDS: Cancer. Morbidity. Lethality. Hospital services. Palliative cares.

Guanche Garcell H, García Arzola E, González Isla J, Salomón Zaldivar E, Pérez, Montero P. Tendencias de la morbilidad y letalidad hospitalaria por cáncer. An Med Interna (Madrid) 2007; 24: 12-14.

\section{INTRODUCCIÓN}

Según el informe mundial sobre el cáncer, su incidencia podría aumentar en un 50\% para el año 2020, con 15 millones de nuevos casos . En el año 2000 los tumores malignos fueron la causa de $12 \%$ de las defunciones que se produjeron en el mundo por todas las causas, de ellos 5,3 millones de hombres y 4,7 millones de mujeres, presentaron tumores malignos.
El informe también revela que el cáncer ha pasado a ser un importante problema de Salud Pública en los países en desarrollo igualando el comportamiento que tiene en los países industrializados, con tendencia al incremento de la incidencia en todos los países y en todas las localizaciones. Ello se relaciona con el envejecimiento de la población, la prevalencia del consumo de tabaco, la alimentación inadecuada, la contaminación atmosférica, al estrés y a la adopción de modos de

Trabajo aceptado: 4 de septiembre de 2006 
vida malsanos. Los gobiernos, los médicos y los educadores sanitarios podrían hacer mucho mas para ayudar a las personas a cambiar su comportamiento con el fin de evitar los cánceres potencialmente prevenibles (1).

Cuba no queda fuera de este marco y la distribución de la mortalidad tiene un patrón comparable al de los países mas desarrollados. Las tasas de muerte por cáncer han tenido una tendencia estable desde los años setenta ocupando el segundo lugar en todos los grupos de edades con excepción del grupo de 1 a 4 años, teniendo una tasa de incidencia ajustada a la población mundial en el año 2002 de 199,6 por 100.000 habitantes y con una mortalidad en el 2004 de 111,16 por 100.000 habitantes (2,3).

El Programa Nacional del Cáncer en Cuba orienta las acciones dirigidas a la educación sanitaria de la población, diagnóstico precoz, tratamiento eficaz y atención al paciente, en todos los niveles del sistema nacional de salud.

El Hospital Universitario Clínico Quirúrgico “Joaquín Albarrán" se ocupa de la atención secundaria de la población adulta del oeste de la capital cubana, y parte de los municipios del centro de la ciudad, por lo que es frecuente ingresar pacientes con cáncer, para diagnóstico, tratamiento oncoespecífico o tratamiento paliativo, lo que nos ha motivado a evaluar la tendencia de la morbilidad y mortalidad hospitalaria por cáncer.

\section{DISEÑO}

Realizamos un estudio de series temporales de los ingresos por cáncer en el Hospital Universitario Clínico Quirúrgico “Joaquín Albarrán” desde 1999 al 2005.

Del Departamento de Registros Médicos se obtuvo la información referida a los ingresos totales del hospital, ingresos por tumores malignos, las defunciones, y las tasas de letalidad (fallecidos por cáncer/ ingresados por cáncer x 100). Se obtuvo información sobre distribución de los ingresos según servicios

De los ingresos ocurridos durante el año 2005 se obtuvo la edad (media y desviación estándar) según sexo, la causa de la admisión, y se precisó si el cáncer se diagnóstico durante esta admisión o fue anterior a la misma.

Toda la información fue analizada utilizando la técnica estadística de análisis de distribución de frecuencias.

\section{RESULTADOS}

En la tabla I podemos observar un sostenido incremento de la proporción de ingresados por cáncer en relación con los ingresos totales de la institución, con la proporción menor de $3 \%$ en el año 1999 y la mayor en el 2005 de un 7,99\%. En relación con las tasas de letalidad observamos un comportamiento irregular con la menor tasa en el 1999 (12,7\%) y la mayor durante el $2001(27,86 \%)$.

Es evidente que la mayoría de los ingresos por cáncer provienen del servicio de medicina interna los que en el periodo estudiado constituyeron el $60,77 \%$, seguidos de los pacientes atendidos en cirugía general $(26,81 \%)$, siendo menos frecuente los pacientes con cáncer en el resto de los servicios institucionales (Tabla II).
Los pacientes ingresados con cáncer durante el 2005 tuvieron edades de 63,55 \pm 15,41 años, la cual fue ligeramente superior en pacientes del sexo masculino $(68,46 \pm 14,10$ años) en relación al sexo femenino (59,68 \pm 15,42 años).

El $44 \%$ de los pacientes se les diagnosticó cáncer durante el ingreso, mientras que el $56 \%$ se les había realizado diagnóstico de su enfermedad previamente. De estos en el $42,1 \%$ el motivo de ingreso fue la necesidad de tratamiento (quirúrgico o quimioterapia antineoplásica) (Fig. 1), mientras el 53,5\% fue admitido por complicaciones propias del proceso maligno (p. ej. vómitos, metástasis, deshidratación, infecciones y otras), entre los cuales el 13,2\% de los ingresos fueron por infecciones, fundamentalmente del tracto respiratorio o urinario y solo el 4,4\% de las admisiones por cáncer fueron para cuidados paliativos.

\section{DISCUSIÓN}

La incidencia poblacional de cáncer en Cuba ha tenido una tendencia ascendente en las últimas décadas, lo cual se refleja en la mayor utilización de servicios de salud por estos enfer$\operatorname{mos}(3,4)$.

En correspondencia con el incremento de la incidencia poblacional del cáncer en Cuba se ha producido un incremento del uso de los servicios de hospitalización por pacientes con estas enfermedades en el periodo analizado (5), lo que tiene un impacto sobre los indicadores de utilización de la cama y los costos del sistema de salud.

El servicio de medicina interna sigue constituyendo el principal servicio relacionado con la atención de estos pacientes, básicamente para diagnóstico de la enfermedad maligna y para tratamiento de las complicaciones. El servicio de oncología posee desde hace varios años un departamento donde se realiza la quimioterapia antineoplásicas de forma ambulatoria, por lo cual los pacientes, en general, no ingresan por esta causa, con la excepción de los que requieren esquemas más prolongados de tratamiento citotóxico.

Por otra parte son menos frecuentes los ingresos de pacientes para cuidados paliativos, los cuales en general deben ser ofrecidos por los equipos locales de salud entrenados en las modalidades terapéuticas que incluyen estos cuidados. Ello es posible potenciarlo más por la oportunidad que ofrece la organización del sistema de salud en Cuba, con un sistema de atención primaria sólidamente relacionado con las instituciones hospitalarias o del nivel secundario.

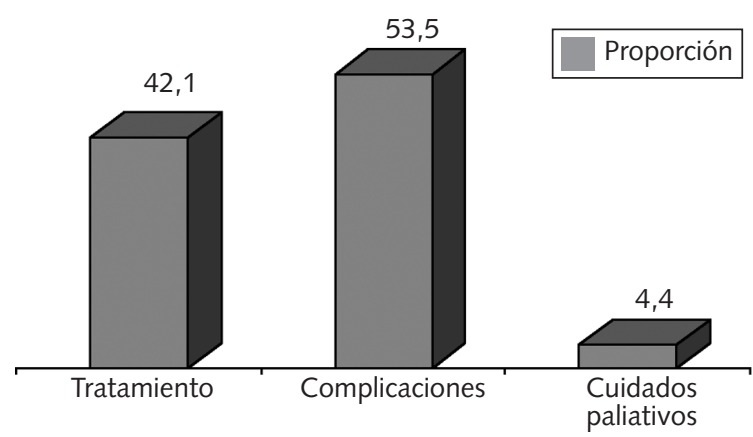

Fig. 1. Proporción de admisiones según causa en pacientes con antecedentes de cáncer ingresados durante el 2005. 
TABLA I

COMPORTAMIENTO DE LOS INGRESOS Y DEFUNCIONES POR CÁNCER. HOSPITAL “JOAQUÍN ALBARRÁN" (1999-2005)

\begin{tabular}{cccccc}
\hline Año & $\begin{array}{c}N^{0} \text { de ingresos } \\
\text { totales }\end{array}$ & $\begin{array}{c}N^{\circ} \text { de ingresos } \\
\text { por cáncer }\end{array}$ & $\begin{array}{c}\text { Proporción de } \\
\text { ingresos por cáncer }\end{array}$ & $\begin{array}{c}N^{\circ} \text { de Fallecidos } \\
\text { por cáncer }\end{array}$ & $\begin{array}{c}\text { Tasa de } \\
\text { letalidad }\end{array}$ \\
\hline 1999 & 14700 & 441 & 3,00 & 56 & 12,70 \\
2000 & 12754 & 494 & 3,87 & 93 & 18,83 \\
2001 & 12347 & 463 & 3,75 & 129 & 27,86 \\
2002 & 11463 & 611 & 5,33 & 119 & 19,48 \\
2003 & 12353 & 662 & 5,36 & 110 & 16,62 \\
2004 & 10529 & 688 & 6,53 & 92 & 13,37 \\
2005 & 9554 & 763 & 7,99 & 127 & 16,64 \\
\hline
\end{tabular}

TABLA II

INGRESOS POR TUMORES MALIGNOS SEGÚN SERVICIO. HOSPITAL “JOAQUÍN ALBARRÁN” (1999-2005)

\begin{tabular}{|c|c|c|c|c|c|c|c|c|c|c|c|c|}
\hline \multirow[t]{2}{*}{ Año } & \multicolumn{2}{|c|}{ Ortopedia } & \multicolumn{2}{|c|}{$O R L$} & \multicolumn{2}{|c|}{ Urología } & \multicolumn{2}{|c|}{ Proctología } & \multicolumn{2}{|c|}{ Cirugía } & \multicolumn{2}{|c|}{ Medicina } \\
\hline & No & $\%$ & No & $\%$ & No & $\%$ & & $\%$ & No & $\%$ & & $\%$ \\
\hline 1999 & 5 & 1,13 & 20 & 4,54 & 16 & 3,63 & 24 & 5,44 & 160 & 36,28 & 216 & 48,98 \\
\hline 2000 & 10 & 2,02 & 24 & 4,86 & 13 & 2,63 & 18 & 3,64 & 122 & 24,70 & 307 & 62,15 \\
\hline 2001 & 7 & 1,51 & 16 & 3,46 & 18 & 3,89 & 20 & 4,32 & 73 & 15,77 & 329 & 71,06 \\
\hline 2002 & 0 & 0,00 & 12 & 1,82 & 14 & 2,12 & 26 & 3,93 & 207 & 31,32 & 402 & 60,82 \\
\hline 2003 & 1 & 0,15 & 9 & 1,36 & 10 & 1,51 & 16 & 2,42 & 195 & 29,46 & 431 & 65,11 \\
\hline 2004 & 8 & 1,16 & 20 & 2,91 & 18 & 2,62 & 25 & 3,63 & 190 & 27,62 & 427 & 62,06 \\
\hline 2005 & 7 & 0,92 & 20 & 2,62 & 13 & 1,70 & 22 & 2,88 & 204 & 26,74 & 497 & 65,14 \\
\hline total & 38 & 0,89 & 121 & 2,82 & 102 & 2,38 & 151 & 3,52 & 1151 & 26,81 & 2609 & 60,77 \\
\hline
\end{tabular}

\section{¿QUÉ IMPLICACIONES TIENE EL COMPORTAMIENTO EN LA MORBILIDAD HOSPITALARIA POR CÁNCER?}

Si consideramos que el empleo de los servicios de hospitalización se está incrementando sostenidamente, quizá en detrimento de la disponibilidad de camas para otros problemas de salud, como las cardiopatías isquémicas o las enfermedades cerebrovasculares que son frecuentes en la población cubana (3), es evidente que el sistema tiene que analizar el problema.

Importante parece ser mirar al futuro, pues, si la actual tendencia se mantiene, en el 2020 más del 15\% de los ingresos pudieran ser por tumores malignos. Para enfrentar está demanda de servicios especializados de salud deberemos modificar las estrategías de atención de estos pacientes, lo cual pudiera incluir:

\section{Bibliografía}

1. Stewart BM, Kleihues P. World Cancer Report. IARC Nonserial Publication, 2003.

2. Dirección Nacional de Estadísticas. Ministerio de Salud Pública de la República de Cuba. http://www.dne.sld.cu/index.htm. Acceso 12 de abril 2006.

3. Anuarios estadísticos. Ministerio de Salud Pública de la República de Cuba. http://www.infomed.sld.cu/servicios/estadisticas/ acceso 8 de
1. Fortalecer los servicios ambulatorios dedicados al diagnóstico rápido y al seguimiento de los pacientes,

2. Crear servicios de hospitalización especializados,

3. Elevar los niveles de capacitación del personal en el diagnóstico y manejo de pacientes con enfermedades malignas,

4. Fortalecer la interrelación con los equipos locales de salud.

El sistema de salud deberá adaptarse a los nuevos tiempos en los que se espera un mayor número de pacientes con enfermedades malignas, por lo cual deberán producirse cambios de la organización de los servicios de salud y de capacitación de los profesionales de la salud que enfrenten las demandas y aseguren la calidad de los servicios de salud.

mayo del 2006

4. Soriano García JL, Galán Álvarez Y, Luaces Álvarez P, Martín García A, Arrebola Suárez J, Carrillo Franco G. Incidencia en cuba del cáncer en la tercera edad. Rev Cubana Oncol 1998; 14: 121-28.

5. Bosetti Cristina; La Vecchia Carlo. Cancer mortality in Latin America: implications for prevention. Rev Panamer Salud Publ 2005; 18 : $1-4$. 